\section{Conjugated polymers optically regulate the fate of endothelial colony-forming cells}

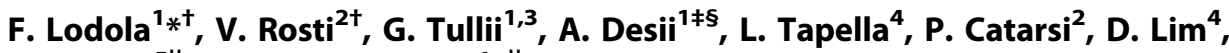 \\ F. Moccia ${ }^{5 \|}$, M. R. Antognazza ${ }^{1} * \|$
}

The control of stem and progenitor cell fate is emerging as a compelling urgency for regenerative medicine. Here, we propose a innovative strategy to gain optical control of endothelial colony-forming cell fate, which represents the only known truly endothelial precursor showing robust in vitro proliferation and overwhelming vessel formation in vivo. We combine conjugated polymers, used as photo-actuators, with the advantages offered by optical stimulation over current electromechanical and chemical stimulation approaches. Light modulation provides unprecedented spatial and temporal resolution, permitting at the same time lower invasiveness and higher selectivity. We demonstrate that polymer-mediated optical excitation induces a robust enhancement of proliferation and lumen formation in vitro. We identify the underlying biophysical pathway as due to light-induced activation of TRPV1 channel. Altogether, our results represent an effective way to induce angiogenesis in vitro, which represents the proof of principle to improve the outcome of autologous cell-based therapy in vivo.

\section{INTRODUCTION}

In recent years, organic semiconductors have emerged as highly promising materials in biotechnology, thanks to several key-enabling features. Differently from silicon-based electronics, they support both electronic and ionic charge transport (1); they can be easily functionalized with specific excitation and sensing capabilities (2-4); and they are solution processable, soft, and conformable (5). They are highly biocompatible, being suitable for in vivo implantation and long-term operation, as recently reported for many different applications, including electrocorticography, precise delivery of neurotransmitters, electrocardiography, deep brain stimulation, and spinal cord injury (6-9). An important, distinctive feature of organic semiconductors is their sensitivity to the visible and near-infrared light. Recently, our and other groups have exploited it for optical modulation of cell electrophysiological activity, by using conjugated polymers and organic molecules as exogenous light-sensitive actuators (10-12). Interesting applications have been reported in the field of artificial visual prosthesis (5), photothermal excitation or inhibition of cellular activity $(13,14)$, and modulation of animal behavior (15).

In this framework, the opportunity to use polymer-based phototransduction mechanisms to regulate the very early stages of living cell development has been very scarcely considered $(16,17)$. The possibility to selectively and precisely regulate a number of cell processes, such as adhesion, differentiation, proliferation, and migration,

\footnotetext{
${ }^{1}$ Center for Nano Science and Technology @PoliMi, Istituto Italiano di Tecnologia, via Pascoli 70/3, 20133 Milano, Italy. ${ }^{2}$ Center for the Study of Myelofibrosis, Laboratory of Biochemistry, Biotechnology and Advanced Diagnosis, IRCCS Policlinico San Matteo Foundation, Pavia, Italy. ${ }^{3}$ Department of Physics, Politecnico di Milano, Piazza L. Da Vinci 32, 20133 Milano, Italy. ${ }^{4}$ Department of Pharmaceutical Sciences, Università del Piemonte Orientale "Amedeo Avogadro," Novara, Italy. ${ }^{5}$ Laboratory of General Physiology, Department of Biology and Biotechnology "Lazzaro Spallanzani," University of Pavia, Pavia, Italy. *Corresponding author. Email: francesco.lodola@iit.it (F.L.); mariarosa.antognazza@iit.it (M.R.A.)

†These authors contributed equally to this work

¥Present address: Dipartimento di Ingegneria Meccanica e Aerospaziale, Politecnico di Torino, Corso Duca degli Abruzzi 24, 10129 Torino, Italy.

§Present address: Smart Bio-Interfaces Italian Institute of Technology, Viale Rinaldo Piaggio 34, 56025 Pontedera (PI), Italy.

||These authors share senior authorship.
}

would be key to regenerative medicine and drug screening. The presently dominant approaches to reliably regulate stem and progenitor cell fate for regenerative purposes mainly rely on the use of chemical cues. However, irreversibility and lack of spatial selectivity represent important limitations of these methods. Whenever targeting in vivo applications, one must face the major, unsolved problem of diffusion of neurotrophic molecules by the conventional intravenous or oral routes. In addition, the therapeutic outcome of autologous cell-based therapy is often impaired by low engraftment, survival, and poor integration of stem cells within the environment of the targeted tissue. Other stimuli, mainly consisting of mechanical and electrical cues, were recently reported to have some notable effects, and recent advances in nanotechnology and material science enabled versatile, robust, and larger-scale modulation of the cell fate. In particular, carbon-based materials and conjugated polymers led to interesting results (18). However, their distinctive visible light absorption was never exploited in optically driven techniques.

Use of light actuation has been proposed either by viral transfer of light-sensitive proteins, by optogenetics tools, or by absorption of endogenously expressed light-sensitive moieties, based on low light-level therapies (19-21). In the first case, interesting results were obtained (22); however, this approach bears all the drawbacks related to the need for viral gene transfer. Photobiomodulation led to interesting outputs as well, but overall efficiency is hampered by the limited absorption of light-responsive molecules endogenously expressed in living cells.

In this work, we propose to couple the use of conjugated polymers with visible light excitation to gain optical control of cell fate. We focus our attention on endothelial progenitor cells (EPCs) and, in particular, on endothelial colony-forming cells (ECFCs), which are currently considered the bona fide best surrogate of EPCs (23). ECFCs are mobilized from the bone marrow and vascular stem cell niche to reconstruct the vascular network destroyed by an ischemic insult and to restore local blood perfusion (24). ECFCs may be easily harvested from peripheral blood, display robust clonogenic potential, exhibit tube-forming capacity in vitro, and generate vessel-like structures in vivo $(24,25)$, thereby representing a promising candidate for autologous cell-based therapy of ischemic disorders (24). Manipulating 
the signaling pathways that drive ECFC proliferation, migration, differentiation, and tubulogenesis could represent a reliable strategy to improve the regenerative outcome of therapeutic angiogenesis in the harsh microenvironment of an ischemic tissue, such as the infarcted heart $(24,25)$. Intracellular $\mathrm{Ca}^{2+}$ signals play a crucial role in stimulating ECFC proliferation and tubulogenesis by promoting the nuclear translocation of the $\mathrm{Ca}^{2+}$-sensitive nuclear transcription factor $\kappa \mathrm{B}(\mathrm{NF}-\kappa \mathrm{B})$ (26-28). It has, therefore, been suggested that intracellular $\mathrm{Ca}^{2+}$ signaling could be targeted to boost the regenerative potential of autologous ECFCs for regenerative purposes (29). For the above-mentioned reasons, ECFCs represent a valuable test bed model for assessing the possibility to exploit the visible light sensitivity of conjugated polymers to gain touchless, optical modulation of cell proliferation and function.

In this framework, we demonstrate that polymer-mediated optical excitation during the first steps of ECFC growth leads to a robust enhancement of both proliferation and tubulogenesis through the optical modulation of the $\mathrm{Ca}^{2+}$-permeable transient receptor potential vanilloid 1 (TRPV1) channel and NF- $\kappa \mathrm{B}-$ mediated gene expression. Our results represent, to the best of our knowledge, the first report on the use of polymer photoexcitation for the in vitro modulation of ECFC fate and function, thereby representing the proof of principle to obtain direct control of progenitor cell fate.

\section{RESULTS}

\section{Realization of poly(3-hexyl-thiophene) polymer/ECFC culture interfaces, viability assay, and optical excitation protocol}

Figure 1A shows a sketch of the bio/polymer interface developed for obtaining optical control of ECFC proliferation and network formation, together with the polymer chemical structure and the optical absorption spectrum. The material of choice for light absorption and phototransduction is a workhorse organic semiconductor, widely used in photovoltaic and photodetection applications, namely, regioregular poly(3-hexyl-thiophene) (P3HT) (6). It is characterized by a broad optical absorption spectrum, in the blue-green visible region, peaking at $520 \mathrm{~nm}$. P3HT outstanding biocompatibility properties have been reported in a number of different systems, both in vitro and in vivo, including astrocytes (30), primary neurons and brain slices (14), and invertebrate models of Hydra vulgaris (15). Chronical implantation of P3HT-based devices in the rat subretinal space did not show substantial inflammatory reactions up to 6 months in vivo (10). Here, polymer thin films (approximate thickness, $150 \mathrm{~nm}$ ) have been deposited by spin coating on top of polished glass substrates, as detailed in Materials and Methods. Both polymer-coated and glass substrates have been thermally sterilized $\left(120^{\circ} \mathrm{C}, 2\right.$ hours), coated with fibronectin, and, lastly, used as light-sensitive and control cell culturing substrates, respectively. ECFCs have been isolated from peripheral blood samples of human volunteers and seeded on top of polymer and glass substrates.

ECFC proliferation on polymer substrates has been primarily assessed in dark conditions at three different time points, namely, 24, 48, and 72 hours after plating (Fig. 1B). Polymer-coated samples, while showing from the very beginning a slightly lower number of cells as compared with control substrates, exhibit a proliferation rate fully similar to cells plated on glass substrates (slope of the linear fitting is $0.034 \pm 0.003, R^{2}=0.99$ and $0.034 \pm 0.005, R^{2}=0.96$ for control and $\mathrm{P} 3 \mathrm{HT}$ polymer samples, respectively).

Once assessed that the P3HT polymer surface represents a nicely biocompatible substrate for ECFC seeding and proliferation in the dark, we moved to investigate the effect of polymer photoexcitation. In more detail, to evaluate the effect of optical stimulation on cell proliferation and network formation, we continuously shined light for the whole temporal window required for cell growth, and we realized an ad hoc system suitable for operation within the cell incubator. The experimental configuration and the excitation protocol are schematically represented in Fig. 1C. Optical excitation is provided by a light-emitting diode (LED) source, with maximum emission wavelength at $525 \mathrm{~nm}$, incident from the substrate side. The choice of the protocol, continuously administered to the cell cultures during early seeding and proliferation stages, has been mainly dictated by the need to avoid overheating effects, with possible negative outcomes on the overall cell culture viability. On the basis of these considerations, we opted for a protocol based on 30-ms excitation pulses, followed by a 70-ms dark condition, at a photoexcitation density of $40 \mathrm{~mW} / \mathrm{cm}^{2}$. The whole protocol is continuously repeated for a minimum of 4 up to 36 hours, depending on the type of functional assay, at controlled temperature $\left(37^{\circ} \mathrm{C}\right)$ and $\mathrm{CO}_{2}$ levels $(5 \%)$.

The temporally precise and spatially localized measurement of the temperature variation upon polymer photoexcitation at the polymer/ cell interface (i.e., within the cell cleft) is not straightforward because
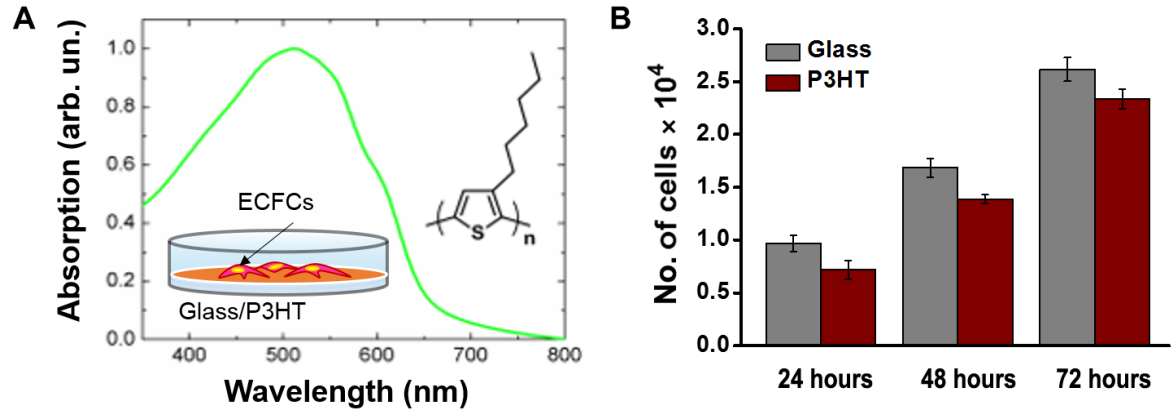

C

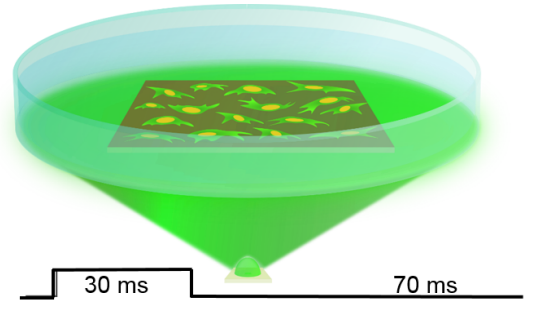

Fig. 1. Polymer devices for optical stimulation of ECFC cultures. (A) P3HT polymer optical absorption spectrum. Insets show the chemical structure of the conjugated polymer and a sketch of the polymer device used for cell optical activation. ECFCs are cultured on top of P3HT thin films, deposited on glass substrates. (B) ECFC viability at fixed time points after plating $\left(24,48\right.$, and 72 hours). Cell cultures were kept in dark conditions at controlled temperature $\left(37^{\circ} \mathrm{C}\right)$ and fixed $\mathrm{CO}_{2}$ levels $(5 \%)$. No statistically significant difference was observed between the glass and polymer substrates at any fixed time point (unpaired Student's $t$ test). (C) Experimental setup and optical excitation protocol for evaluation of polymer-mediated cell photoexcitation effects on cell fate. Polymer and control samples are positioned within a sterilized, home-designed petri holder. Light scattering effects are completely screened. The geometry and the photoexcitation protocol have been implemented to minimize overheating effects and to keep the overall extracellular bath temperature fairly unaltered. Thirty-millisecond-long green light pulses are followed by $70 \mathrm{~ms}$ in dark condition. 
it requires the use of localized, submicrometer probes with a fast response time. However, according to the heat diffusion equation, we expect that dissipation occurs within a few milliseconds, following exponential decrease dynamics (14). Moreover, we used the wellknown method of the calibrated pipette (31) to characterize the temperature variation dynamics within the extracellular bath volume, defined by the cylinder with the base area corresponding to the light spot size and the height of about $1 \mu \mathrm{m}$. This choice is a good approximation of the overall volume occupied by a single ECFC cell; thus, it provides a realistic estimation of the average heating experienced by the cell (fig. S1A). We observe that temperature variation closely follows short optical pulse dynamics, reaching a maximum temperature at the end of the 30-ms illumination period, quickly followed by an almost complete thermal relaxation to the basal temperature during the 70-ms-long dark period. We conclude that our polymer-based system provides a highly spatially and temporally resolved method for optical excitation, making it possible, in perspective, to selectively target single cells and even cell subcompartments. Upon prolonged illumination (hours), one should also consider possible overheating effects of the whole extracellular medium volume. The average temperature of the bath for the entire duration of the long-term experiment was measured by a thermocouple immersed in the medium. Data show that an equilibrium situation is established after 5 hours and that the absolute temperature of the bath is increased by about $1.5^{\circ}$ (fig. S1B). The adopted prolonged excitation protocol does not negatively affect overall cell culture viability (see below).

\section{Polymer optical excitation drives ECFC proliferation and in vitro tubulogenesis}

Figure 2 reports specific effects mediated by P3HT substrates and visible light stimulation on ECFC proliferation. ECFCs were plated in the presence of EGM-2 medium to facilitate the adhesion to the substrate. After 12 hours, the medium was switched to EBM-2 supplemented with $2 \%$ fetal bovine serum, and the cells were subjected to the long-term lighting protocol for 36 hours at controlled temperature $\left(37^{\circ} \mathrm{C}\right)$ and $\mathrm{CO}_{2}$ levels $(5 \%)$. Under these conditions, ECFCs seeded on P3HT and subjected to light stimulation undergo a significant increase in proliferation rate, as compared with the control condition, i.e., to cells also seeded on P3HT polymer substrates but kept in dark conditions for the whole duration of the experiment $(+158 \%$ versus P3HT dark; $P<0.05)$. No statistically significant difference in proliferation was observed among cells seeded on glass, whether they were subjected to optical excitation or not (Fig. 2A).

Recent evidence demonstrated an interesting correlation between processes key to ECFC vascular regeneration, including proliferation and network formation, and activation of TRPV1 channels, which are expected to be endogenously expressed in ECFCs (32). In addition, we recently reported that polymer photoexcitation leads to selective TRPV1 activation in transfected human embryonic kidney (HEK) cell models (33). Therefore, we were prompted to evaluate whether the increase in cell proliferation is distinctively determined by a polymer-mediated photoactivation of the TRPV1 channel. To this goal, we preliminarily checked the actual expression of the TRPV1 channel in the ECFC models by carrying out electrophysiology experiments in patch-clamp configuration. Methods and results are extensively discussed in the Supplementary Materials (fig. S2 and related description). Briefly, the expression of the TRPV1 channel was confirmed, as well as the capability to selectively excite its activity through localized polymer excitation at high optical power density. To establish whether the TRPV1 channel also has a role in the observed increase in cell proliferation upon polymer excitation, we performed the experiments under light illumination upon administration of a highly specific TRPV1 antagonist [capsazepine (CPZ), $10 \mu \mathrm{M}$ ], an aspecific TRPV channel inhibitor [ruthenium red (RR), $10 \mu \mathrm{M}$ ], and a selective antagonist of a different temperature-sensitive channel, TRPV4, which is also endogenously expressed in ECFCs (RN-1734, $20 \mu \mathrm{M})$ (34) (Fig. 2B). TRPV1 inactivation by CPZ and RR results in a relative, strong reduction in cell proliferation by 51 and $30 \%$, respectively, as compared with untreated cells. Conversely, in the case of RN-1734 treatment, the proliferation increase due to polymer photoexcitation is completely unaltered.

As mentioned earlier, intracellular $\mathrm{Ca}^{2+}$ signaling has been reported to drive ECFC proliferation $(26,28)$. To further investigate whether TRPV1-mediated extracellular $\mathrm{Ca}^{2+}$ entry mediates the proangiogenic response to light illumination, we pretreated ECFCs with [1,2-Bis(2-aminophenoxy)ethane-N,N,N', $\mathrm{N}^{\prime}$-tetraacetic acid tetrakis(acetoxymethyl ester) BAPTA-AM] $(30 \mu \mathrm{M})$, a membranepermeable buffer of intracellular $\mathrm{Ca}^{2+}$ levels $(26,28)$. BAPTA-AM is
A

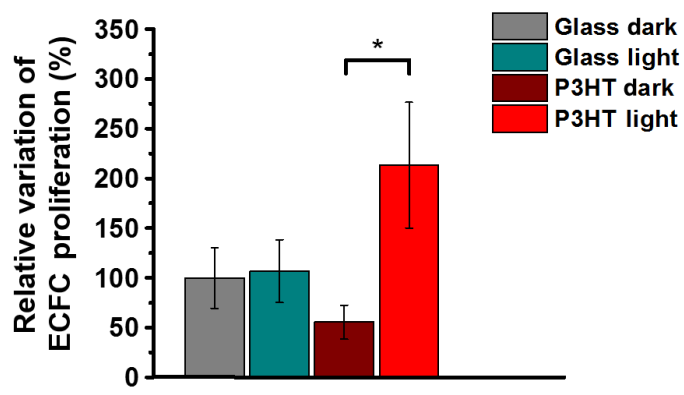

B

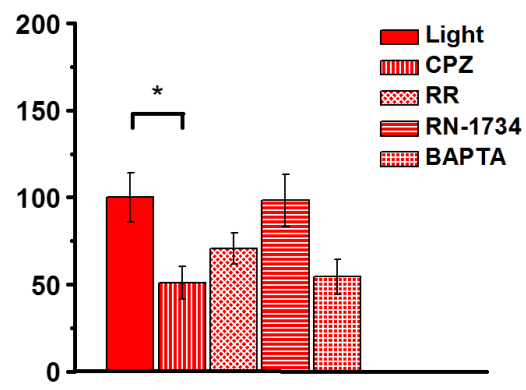

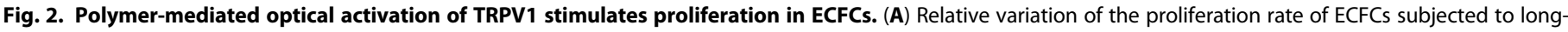

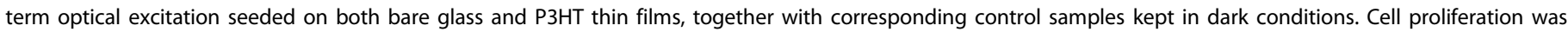

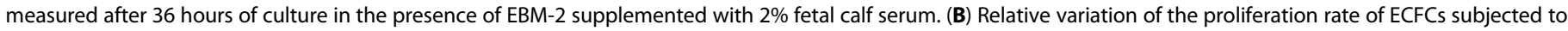

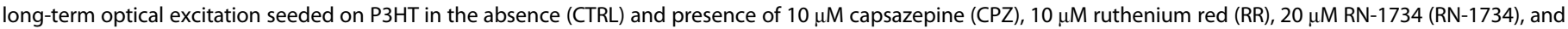

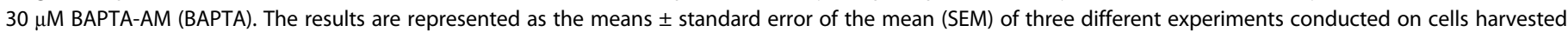

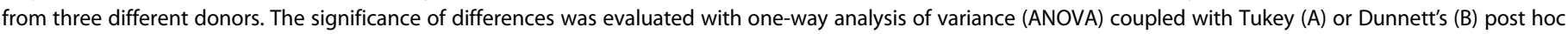
test. ${ }^{*} P<0.05$. 
widely used to prevent the increase in intracellular $\mathrm{Ca}^{2+}$ concentration $\left(\left[\mathrm{Ca}^{2+}\right]_{\mathrm{i}}\right)$ induced by extracellular stimuli and inhibits the downstream $\mathrm{Ca}^{2+}$-dependent processes. For instance, BAPTA-AM represents the most suitable tool to prevent the activation of $\mathrm{Ca}^{2+}$-sensitive decoders residing within tens of nanometers from the inner pore of plasmalemmal Ca ${ }^{2+}$ channels (35). It was recently reported that, in the absence of $\mathrm{Ca}^{2+}$-mobilizing growth factors, it does not impair the low rate of ECFC growth (27). Here, however, BAPTA-AM clearly reduced the light-driven proliferation increase, thus confirming that TRPV1 stimulates ECFCs through an increase in $\left[\mathrm{Ca}^{2+}\right]_{\mathrm{i}}$ (Fig. $2 \mathrm{~B}$ ).

We further examined the physiological outcome of chronic light stimulation by carrying out a tube formation assay within an extracellular matrix protein-based scaffold, which is a surrogate of the basement membrane extracellular matrix. This assay recapitulates many steps of the angiogenic process, including adhesion, migration, protease activity, and tubule formation $(27,28)$. ECFCs were plated in the presence of EBM- 2 medium supplemented with $2 \%$ fetal calf serum and subjected to the long-term lighting protocol for 8 hours at controlled levels of temperature and $\mathrm{CO}_{2}$. Control experiments carried out in dark conditions, either onto glass (see Fig. 3A for a representative optical image) or onto polymer substrates (Fig. 3C), as well as control experiments carried out upon photoexcitation of cells seeded on glass substrates (Fig. 3B), do not show remarkable differences. Conversely, ECFC cultures subjected to polymer-mediated optical excitation clearly tend to assemble into an extended bidimensional capillary-like network (Fig. 3D). Cell cultures were monitored up to 24 hours after illumination onset, but results were comparable to observations reported here, after 8 hours of illumination. This qualitative observation is fully confirmed by quantitative morphological analysis (27). As depicted in the sketch of Fig. 3E, we quantitatively evaluated the main features typical of the capillary-like network formation and, in particular, the number of master segments (Fig. 3F), master junctions (Fig. 3G), and meshes (Fig. $3 \mathrm{H}$ ). In all cases, a notable, statistically relevant difference is observed between cells subjected to polymer-mediated optical excitation and controls. Within the same considered temporal window, the combined use of polymer substrates and visible light stimuli does not lead to sizable toxicity effects or delays in cell proliferation. Conversely, it leads to enhanced cell proliferation (Fig. 2) and allows the achievement of the formation of a more extended and mature tubular network (Fig. 3).

As evidenced for the proliferation rate, the TRPV1 channel activation emerges to play also a fundamental role in tubulogenesis (Fig. 4). The TRPV1 pharmacological blockade with the specific inhibitor CPZ deterministically leads to a marked reduction in network formation (Fig. 4A). Upon CPZ administration, a statistically significant decrease in the relative variation of the number of master segments (Fig. 4E), master junctions (Fig. 4F), and meshes (Fig. 4G) is observed. In line with the results shown in Figs. 2 and 3, RR administration resulted in a less marked but still sizable reduction in the tubular network (Fig. 4, B and E to G), probably due to the minor specificity toward TRPV1, while the protubular effect of light remained fully unaltered in the presence of the TRPV4 inhibitor RN-1734 (Fig. 4, C and E to G). Notably, the treatment with BAPTA-AM $(30 \mu \mathrm{M})$, which affected ECFC proliferation, was able to prevent also in vitro tubulogenesis, thus corroborating the key role of intracellular $\mathrm{Ca}^{2+}$ signaling in the proangiogenic response to light illumination (Fig. 4, D and E to G). Control measurements carried out in dark conditions on polymer substrates upon the considered pharmacological treatments do not show any relevant effect (fig. S4, A to C). Overall, this evidence supports the notion that TRPV1 stimulates ECFC proliferation and network formation and demonstrates that optical excitation, properly mediated by biocompatible polymer substrates, positively affects ECFC fate by spatially and temporally selective activation of the TRPV1 channel.
A

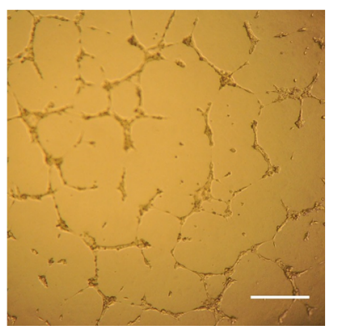

E

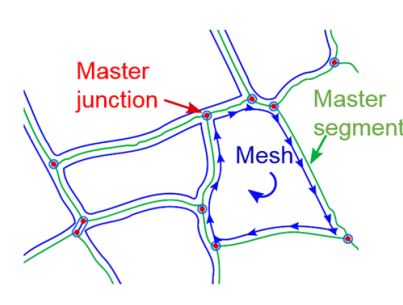

B

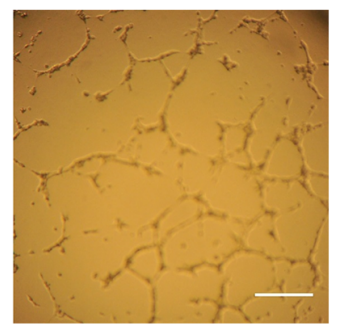

$\mathbf{F}$

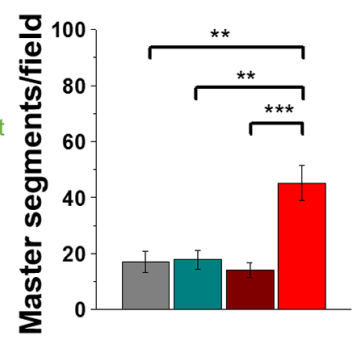

C

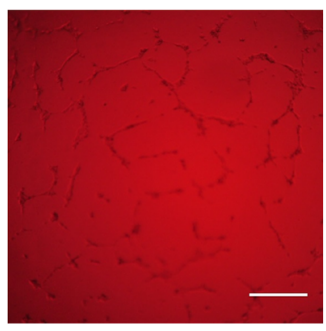

G

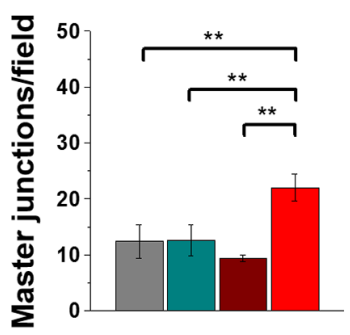

D

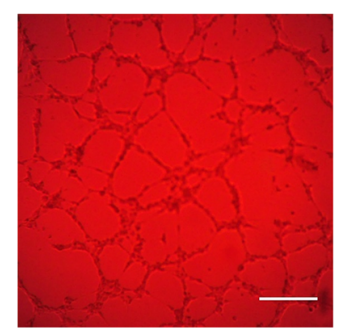

H

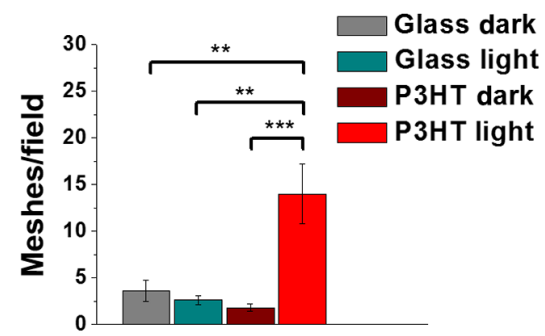

Fig. 3. Light-induced photoexcitation stimulates tubulogenesis in ECFC cultures. (A to D) Representative images of in vitro tubular networks of ECFCs subjected to long-term optical excitation seeded on both bare glass and $\mathrm{P} 3 \mathrm{HT}$, as well as on corresponding control samples in dark conditions. Cultures were observed up to 24 hours, but their appearance did not substantially change after pictures were taken after 8-hour culture. Scale bars, $250 \mu \mathrm{m}$. (E) Sketch representing the main features typical of the capillary-like network that were considered for the topologic analysis. Number of master segments $(\mathbf{F})$, master junctions $(\mathbf{G})$, and meshes $(\mathbf{H})$ analyzed in the different conditions. The results are represented as the means \pm SEM of three different experiments conducted on cells harvested from three different donors. The significance of differences was evaluated with one-way ANOVA coupled with Tukey post hoc test. ${ }^{* *} P<0.01$ and ${ }^{* * *} P<0.001$. 
A

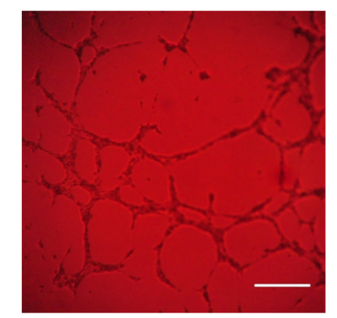

E

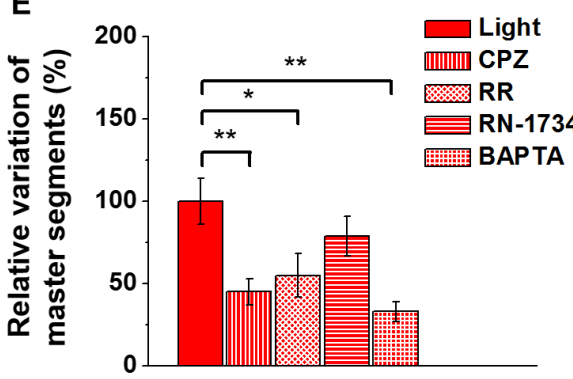

B

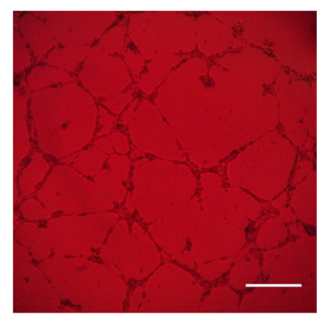

$\mathbf{F}$

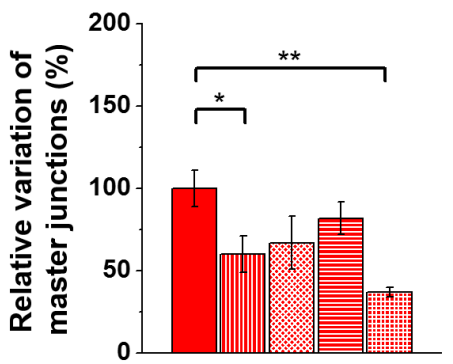

D

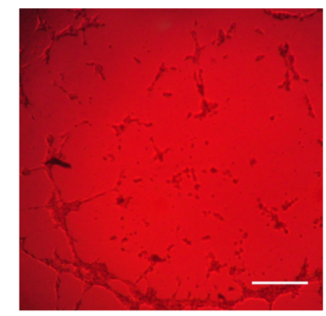

G

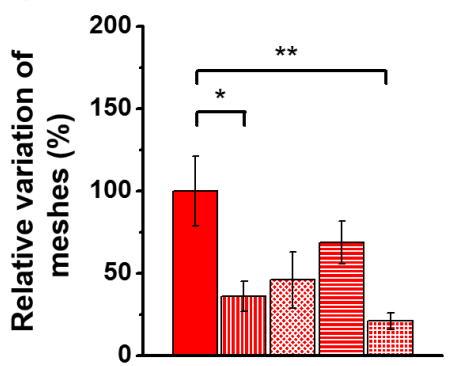

Fig. 4. Light-induced TRPV1 activation promotes in vitro tubulogenesis in ECFC cultures. (A to D) Representative optical images of in vitro tubular network of ECFCs subjected to long-term optical excitation seeded either on bare glass or on P3HT thin films and treated respectively with CPZ (A), RR (B), RN-1734 (C), and BAPTAAM (D). Scale bars, $250 \mu \mathrm{m}$. (E to G) Relative variation of number of master segments (E), master junctions (F), and meshes (G) of ECFCs subjected to long-term optical excitation seeded on P3HT in the absence [control (CTRL)] and presence of $10 \mu \mathrm{M} \mathrm{CPZ,} 10 \mu \mathrm{M}$ RR, $20 \mu \mathrm{M}$ RN-1734 (RN-1734), and $30 \mu \mathrm{M}$ BAPTA-AM (BAPTA). The results are represented as the means \pm SEM of three different experiments conducted on cells harvested from three different donors. The significance of differences was evaluated with one-way ANOVA coupled with Dunnett's post hoc test. ${ }^{*} P<0.05$ and ${ }^{* *} P<0.01$.

\section{Phototransduction mechanism}

We now turn our attention to elucidating the possible mechanisms leading to optically enhanced tubulogenesis, through TRPV1 channel activation, upon prolonged polymer excitation.

Reliable optical modulation of the cell activity mediated by polymer photoexcitation has been reported in several, previous reports, both in vitro, at the level of single cells, and in vivo, at the level of the whole animal, as evidenced by behavioral studies on both invertebrate and vertebrate models. Three different photostimulation mechanisms, active at the polymer/cell interface, have been proposed so far. These include (i) the creation of an interface capacitance, i.e., of a localized electric field, possibly affecting the cell membrane potential (11); (ii) photothermal processes, establishing a localized temperature increase upon polymer photoexcitation $(13,36)$; and (iii) photoelectrochemical reactions, mainly oxygen reduction processes, leading to a local variation of extracellular and/or intracellular $\mathrm{pH}$ (33) and sizable production of reactive oxygen species (ROS), at a nontoxic concentration, and intracellular calcium modulation (37).

In electrophysiological experiments, carried out at a photoexcitation density higher than the one used in "chronic" stimulation by about two orders of magnitude, we clearly observe TRPV1 excitation, corresponding however to a small variation of the cell membrane potential, in the order of a few millivolts (Supplementary Materials). Thus, upon much lower light intensity, the effects of either direct photothermal channel activation and of photocapacitive charging are expected to be negligible. To further corroborate this hypothesis, we carry out control experiments aimed at disentangling photoelectrical from photothermal transduction processes.

First, we use a different material as a cell-seeding substrate, characterized by optical absorption and heat conductivity similar to the ones typical of P3HT (13) but fully electrically inert (i.e., unable to sustain electronic charge generation upon photoexcitation). The material of choice is a photoresist (MicroPosit S1813). S1813 thin films are realized by spin coating, and deposition parameters are optimized to obtain optical absorbance values similar to the semiconducting polymer samples at the considered excitation wavelength. The capability of photoresist substrates to sustain ECFC proliferation was successfully assessed in a control measurement, obtaining fully comparable results with respect to the P3HT substrates (Fig. 5A). The functional effect eventually driven by photoresist optical excitation on tubulogenesis was then investigated by using the same experimental conditions and analysis technique previously adopted for polymer and glass substrates (Fig. 5B). Data show that long-term photoresist excitation does not lead to sizable enhancement of the cellular network formation, thus pointing out that a purely photothermal effect does not play a major role in boosting the tubulogenesis process at variance with semiconducting polymer substrates. In a complementary experiment, we directly assessed the occurrence of photoelectrochemical reactions at the polymer/extracellular bath interface by measuring ROS production. We previously demonstrated that $\mathrm{P} 3 \mathrm{HT}$ polymer thin films exposed to saline electrolytes sustain efficient light-triggered charge generation and charge transfer processes, giving rise to photoelectrochemical reactions $(38,39)$. Moreover, we also reported that $\mathrm{P} 3 \mathrm{HT}$ nanoparticles are efficiently internalized within the cytosol of secondary line cell models (HEK-293) and that their photoexcitation leads to the production of ROS and subsequent intracellular calcium modulation $(15,37)$. However, the actual capability to sustain photoelectrochemical reactions in the specific experimental conditions used in this work (polymer film deposition conditions, sterilization process, prolonged exposure to specific cellular growth medium in an incubating environment, prolonged exposure to a light excitation protocol, light wavelength, pulses duty cycle, and power density) was never assessed. In particular, direct measurement of intracellular ROS was never carried out in the presence of polymer thin films. 
A

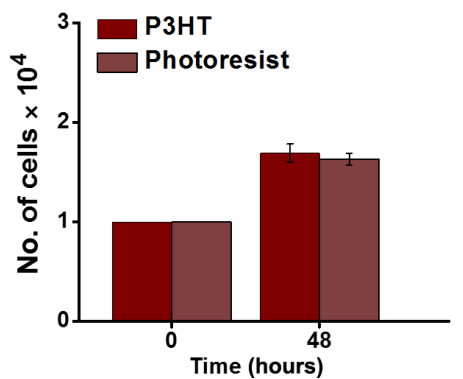

B

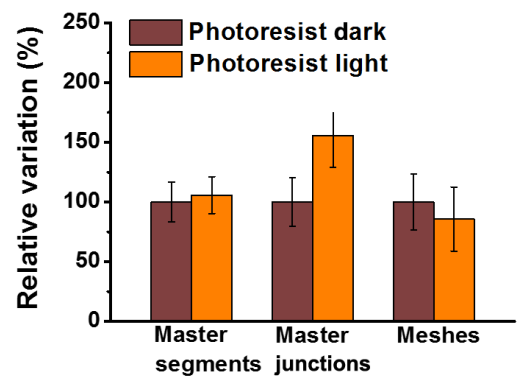

C

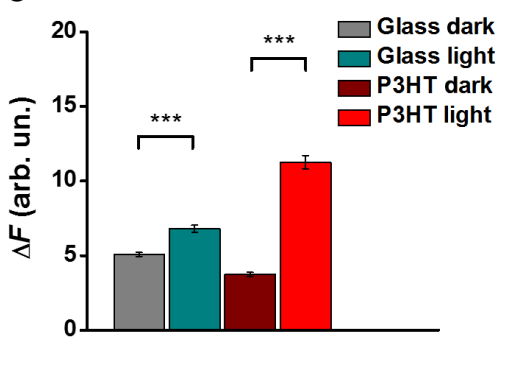

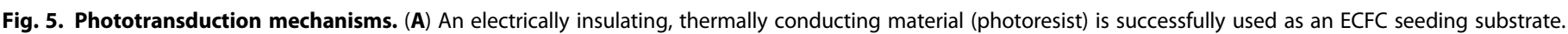

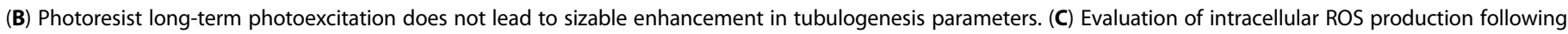

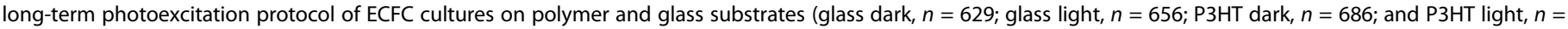

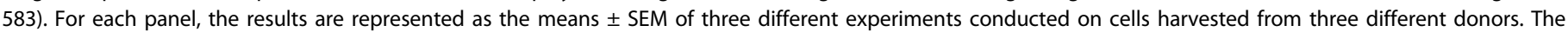
significance of differences was evaluated with unpaired Student's $t$ test (A and B) or one-way ANOVA coupled with Tukey post hoc test (C). ${ }^{* * *} P<0.001$.

To this goal, we realized ECFC cultures on top of polymer and glass control substrates, and we exposed them to the same optical stimulation protocol previously used in the tubulogenesis assay. ROS production was then evaluated by means of a fluorescence experiment based on the use of the well-known ROS probe $2^{\prime}, 7^{\prime}$-dichlorodihydrofluorescein diacetate $\left(\mathrm{H}_{2} \mathrm{DCF}-\mathrm{DA}\right)$ (Fig. $\left.5 \mathrm{C}\right)$. Results show that light induces an increase in ROS production both on glass and polymer substrates. Relative percentage variation amounts to +34 and $+200 \%$, respectively, thus pointing out that polymer surface photocatalytic activity plays a major role in the phototransduction phenomenon.

Altogether, data in Fig. 5 indicate that photoelectrochemical reactions induced by light at the interface between the organic semiconducting polymer and the extracellular bath play a key role in triggering the observed enhancement in cell network formation through indirect activation of the TRPV1 channel. The occurrence of faradaic phenomena at the polymer/bath interface may give rise to material degradation effects. The photostability of the polymer substrates was carefully checked by optical absorption, photoluminescence, and Raman spectra measurements. By treating the samples with the same experimental protocol used for cell tubulogenesis assays (photoexcitation density, pulses frequency, overall exposure duration, temperature, and humidity levels), no sign of irreversible polymer degradation was observed, as compared with nonilluminated samples (fig. S5).

\section{Long-term optical excitation recruits NF-кB and induces the expression of proangiogenic genes}

The $\mathrm{Ca}^{2+}$-sensitive transcription factor NF- $\kappa \mathrm{B}$ might provide the missing link between the influx of $\mathrm{Ca}^{2+}$ through TRPV1 and the increase in proliferation and tubulogenesis observed in ECFCs upon photostimulation (26). We therefore monitored the nuclear translocation of the cytoplasmic p $65 \mathrm{NF}-\kappa \mathrm{B}$ subunit via immunofluorescence staining and mRNA levels of a number of genes induced during tubulogenesis in an NF- $\mathrm{BB}$-dependent manner $(26,40)$ (Fig. 6). Our data indicate that ECFCs seeded on polymer and subjected to light stimulation have a significantly enhanced p $65 \mathrm{NF}-\kappa \mathrm{B}$ nuclear translocation compared with the control conditions consisting of cells also seeded on P3HT but kept in dark conditions (+35\% versus P3HT dark; $P<$ 0.05 ; Fig. $6, A$ and B), and seeded on bare glass ( $+28 \%$ versus glass dark; $P<0.05$; Fig. 6B). No differences were observed between samples seeded on glass, whether they were subjected to optical excitation or not (fig. S6).

In addition, we have checked the expression of nine genes whose expression is known to be induced in endothelial cells during tubulogenesis/ angiogenesis in an NF- $\mathrm{KB}$-dependent manner. We considered intercellular adhesion molecule 1 (ICAM1); vascular adhesion molecule 1 (VCAM1); selectin E (SELE), matrix metalloproteinases (MMPs) 1, 2, and 9; vascular endothelial growth factor A (VEGFA); cyclooxygenase 2 (COX2, PTGS2); and cyclin D1 (CCND1) (40). Of these, five are significantly up-regulated by light exposure in cells grown on P3HT substrates, namely, ICAM1 (+90\% versus P3HT dark; $P<$ 0.05), SELE $(+1119 \% ; P<0.01)$, MMP1 $(+242 \% ; P<0.01)$, MMP2 $(+467 \% ; P<0.05)$, and MMP9 (+458\%; $P<0.05)$ (Fig. 6C). Conversely, VCAM1, VEGFA, PTGS2, and CCND1 do not show relevant variation upon light stimulation (fig. S7A). Light excitation on cells grown on bare glass substrates does not show any significant effect as compared with control samples in dark conditions (fig. S7B).

\section{DISCUSSION}

Therapeutic angiogenesis via autologous EPC transplantation represents a promising strategy to preserve or, at least, partially restore cardiac function after myocardial infarction $(24,41)$. Nevertheless, the regenerative outcome of EPC-based therapies in preclinical studies was rather disappointing and did not lead to sufficient neovascularization of the ischemic heart (41). This led to the proposal to boost their angiogenic activity by using emerging technologies, including tissue engineering of vascular niches, pharmacological preconditioning, or genetic and epigenetic reprogramming (42). ECFCs are regarded among the most suitable EPC subtypes to induce therapeutic angiogenesis and cardiac regeneration due to their high clonal proliferative potential and ability to assemble into capillary-like structures $(23,24)$. In addition, they can be easily isolated and expanded from the peripheral blood of patients and healthy donors. It has recently been suggested that their angiogenic activity could be boosted by targeting the intracellular $\mathrm{Ca}^{2+}$ toolkit (29). Here, we target ECFCs by adopting a fully different approach, i.e., by exploiting visible light as a modulation trigger and by the use of a thiophene-based conjugated polymer as the exogenous, lightresponsive actuator. We demonstrate that photoexcitation of the organic material deterministically leads to robustly enhanced proliferation and tubulogenesis. Pharmacological assays, supported by electrophysiology experiments, allow the identification of TRPV1 selective excitation as a key player in the molecular pathway leading to macroscopic outcomes, as observed by quantitative analysis of the angiogenic response.

All data unambiguously show that polymer photoexcitation leads to selective activation of the TRPV1 channel, which has recently 
A

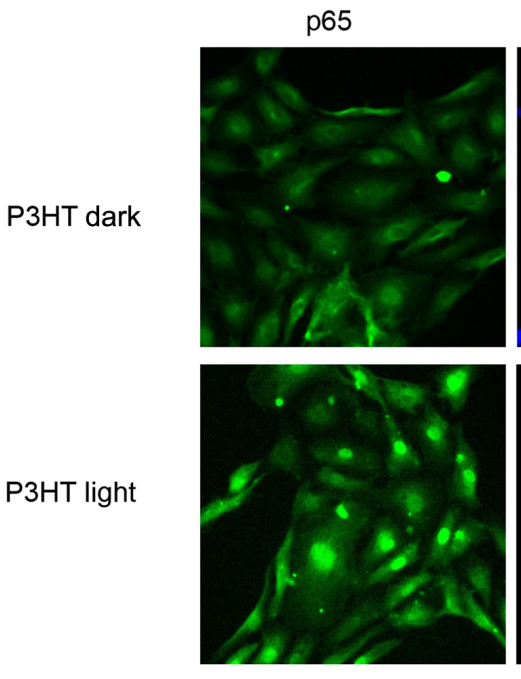

B

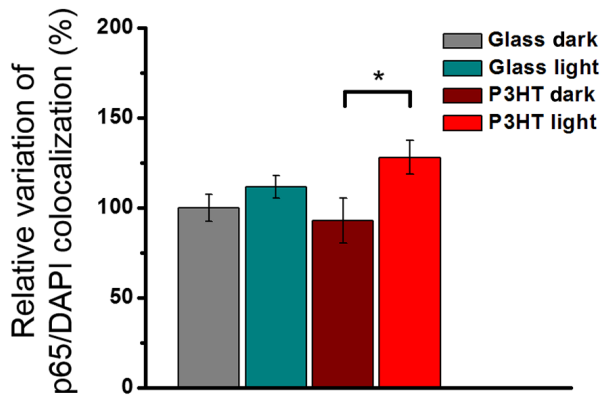

DAPI
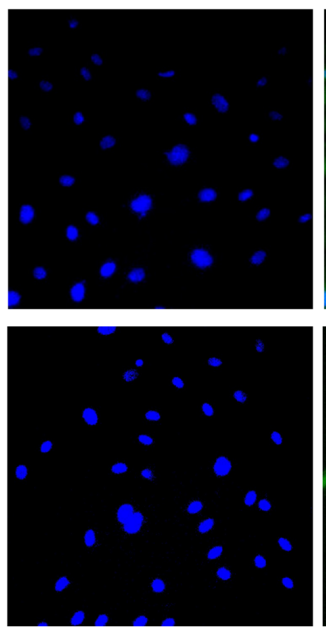

C

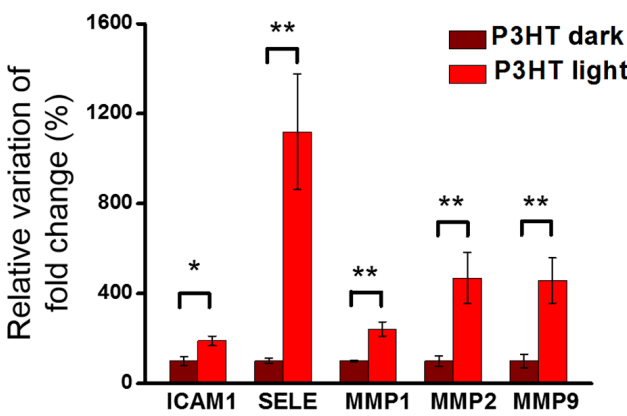

Fig. 6. Light-induced photostimulation promotes p65 NF-kB nuclear translocation and induces the expression of proangiogenic genes in ECFCs. ECFCs seeded on $\mathrm{P} 3 \mathrm{HT}$ samples and glass controls are subjected to long-term photostimulation protocol. Corresponding control samples are kept in dark conditions. After photostimulation, p65 NF- $\mathrm{\kappa B}$ nuclear translocation (A and B) and mRNA levels of tubulogenic/angiogenic genes that have been shown to be activated downstream of NF- $\mathrm{kB}$ (C) are evaluated. (A) Representative images of immunofluorescence staining showing p65 NF-kB (green) nuclear translocation. Cell nuclei are detected by 4',6-diamidino-2phenylindole (DAPI) (blue). Scale bars, $50 \mu \mathrm{m}$. (B) Quantitative evaluation of p65 NF- $\kappa B$ nuclear translocation, as evidenced by colocalization experiments. Results are expressed as means \pm SEM of the relative percentage of p65 nuclei-positively stained cells to the total number of cells (glass dark, $n=151 ;$ glass light, $n=125 ; \mathrm{P} 3 \mathrm{HT}$ dark, $n=147$; and P3HT light, $n=159$ ). Ten fields per condition are analyzed. Data are obtained from two different experiments conducted on cells harvested from two different donors. (C) mRNA levels of intercellular adhesion molecule 1 (ICAM1), selectin E (SELE), and matrix metalloproteinases (MMP1, MMP2, and MMP9) are quantified by real-time polymerase chain reaction (PCR). Data are expressed as means \pm SEM of percentage variation with respect to cells grown in the dark $(n=6)$. The significance of differences was evaluated with unpaired Student's $t$ test $(C)$ or one-way ANOVA coupled with Tukey post hoc test (B). ${ }^{*} P<0.05$ and ${ }^{* *} P<0.01$.

been shown to be expressed and drive angiogenesis in human ECFCs (32). TRPV1 is a polymodal $\mathrm{Ca}^{2+}$-permeable channel that integrates multiple chemical and physical cues to sense major changes in the local microenvironment of most mammalian cells (43). TRPV1 is activated by either noxious heat $\left(>42^{\circ} \mathrm{C}\right)$ and acidic solutions $(\mathrm{pH}<6.5)$, whereas mild acidification ( $\mathrm{pH}$ 6.3) of the extracellular milieus sensitizes TRPV1 to heat stimulation and results in channel activation at temperature thresholds $\left(30^{\circ}\right.$ to $\left.32^{\circ} \mathrm{C}\right)$ well below the normal one (43). ROS production is also expected to further contribute to TRPV1 activation, as previously reported in mouse coronary endothelial cells (44), in which hydrogen peroxide elicits a depolarizing inward current at negative holding potentials. Likewise, ROS may stimulate TRPV1 to depolarize the membrane potential, thereby triggering trains of action potentials in airway C fibers $(45,46)$.

On the basis of measurements carried out in cells seeded on the photoresist substrate, as well as on direct evaluation of a limited, local temperature increase upon light stimuli during the long-term photoexcitation protocol, we infer that the excitation of the TRPV1 channel through direct photothermal transduction is not the predominant process leading to enhanced tubulogenesis.

We have previously demonstrated that polymer photoexcitation leads to generation of faradaic current, to electron transfer reactions at the polymer/electrolyte interface, and to sizable intracellular enhancement of ROS $(37,38)$. Briefly, optical excitation of P3HT polymer thin films leads to photoexcited species (Eq. 1), namely, singlets and charge states, which react with the oxygen dissolved in the cell medium, thus reducing oxygen (Eq. 2)

$$
\begin{gathered}
\mathrm{P} 3 \mathrm{HT}+\mathrm{h} v \rightarrow \mathrm{P} \mathrm{HT}^{*} \\
\mathrm{P} \mathrm{HT}^{*}+\mathrm{O}_{2} \rightarrow \mathrm{P} \mathrm{HT}^{+}+\mathrm{O}_{2}^{-}
\end{gathered}
$$

The superoxide further evolves, leading to the generation of different ROS and, lastly, ending up with hydrogen peroxide production. It has been reported that extracellular $\mathrm{H}_{2} \mathrm{O}_{2}$ can cross the plasma membrane 
through aquaporin AQP3, thereby triggering intracellular ROS signaling $(47,48)$. In line with our previous results, we have demonstrated here that intracellular ROS enhancement does occur in ECFCs upon photoexcitation of polymer thin films, thus contributing to TRPV1 activation.

Altogether, the evidence supports the hypothesis of a transduction mechanism mainly governed by photoelectrochemical reactions. Moreover, these same observations could explain why TRPV4, which is also expressed in ECFCs (34), is not sensitive to optical modulation. Although TRPV4 is activated by moderate heat $\left(24^{\circ}\right.$ to $\left.38^{\circ} \mathrm{C}\right)$, it is supposed to be inhibited by local $\mathrm{pH}$ variation, although this is still a matter of debate $(49,50)$.

On the one hand, the role attributed in the phototransduction mechanism to the capability of the polymer to generate and transport electronic charges, as well as to its photocatalytic activity in an aqueous environment, clearly implies the need for a biocompatible, visible light-responsive, semiconducting material. This excludes any possible implementation of the reported technique by using a thermally conducting, electrically insulating plastic substrate. Suitable cellseeding materials have to be selected and developed within the wide arena of organic semiconducting polymers. On the other hand, the key role played by ROS raises additional issues about material photostability, cell viability, and overall safety and reliability of the technique. We extensively verified that the main polymer optoelectronic properties are not substantially altered by the exposure to light and to incubating conditions. From the biological point of view, it is very well known that high ROS levels can induce highly toxic effects and, finally, lead to cell death. We notice, however, that the established photoactivation protocol (illuminator geometry and air flow, light photoexcitation density, duty cycle, and repetition rate) has been implemented to avoid any detrimental effect. Accordingly, no toxicity effects were detected for the overall duration of the experiments, as proven by the robust increase in ECFC proliferation and tubulogenesis exposed to light. This observation is consistent with the emerging notion that appropriate ROS levels can exert a signaling role and control angiogenesis in endothelial cells (51).

The biophysical mechanisms whereby the photoactivation of TRPV1 stimulates in vitro angiogenesis in ECFCs deserve a more detailed discussion as well. Earlier work showed that TRPV1 stimulates proliferation and tube formation in vascular endothelial cells by mediating extracellular $\mathrm{Ca}^{2+}$ entry. The following increase in intracellular $\mathrm{Ca}^{2+}$ concentration $\left(\left[\mathrm{Ca}^{2+}\right]_{\mathrm{i}}\right)$ leads to the recruitment of several downstream $\mathrm{Ca}^{2+}$-dependent decoders, such as endothelial nitric oxide synthase and $\mathrm{Ca}^{2+} /$ calmodulin-dependent protein kinase II (CaMKII) (52). Recently, TRPV1 was found to induce also proliferation and tube formation in ECFCs by mediating the uptake of the endocannabinoid anandamide (32). This study, however, did not investigate whether TRPV1 activation was per se able to stimulate ECFCs by engaging $\mathrm{Ca}^{2+}$-dependent pathways. Intracellular $\mathrm{Ca}^{2+}$ signaling is a crucial determinant of ECFC fate and behavior (26-28). Accordingly, light-induced ECFC proliferation and tube formation were markedly reduced by the pharmacological blockade of TRPV1-mediated $\mathrm{Ca}^{2+}$ entry with $\mathrm{CPZ}$ and $\mathrm{RR}$ and by preventing the subsequent increase in $\left[\mathrm{Ca}^{2+}\right]_{\mathrm{i}}$ with BAPTA-AM. This finding endorses the view that optical excitation stimulates ECFCs through TRPV1-mediated extracellular $\mathrm{Ca}^{2+}$ entry, and we suggest here that this occurs via downstream activation of transcriptional factor NF- $\kappa \mathrm{B}$. NF- $\kappa \mathrm{B}$ has previously been shown to stimulate cell proliferation and tubulogenesis in endothelial cells $(53,54)$ and in hepatocytes (55). Our group has shown that NF- $\kappa \mathrm{B}$ triggers the transcriptional program underlying the angiogenic response to extracellular $\mathrm{Ca}^{2+}$ entry in ECFCs (26). Moreover, NF- $\kappa \mathrm{B}$ activation in response to extracellular stimulation and $\mathrm{Ca}^{2+}$ entry through TRPV1 has also been demonstrated $(56,57)$. Under resting conditions, NF- $\mathrm{KB}$ is retained in the cytoplasm by the

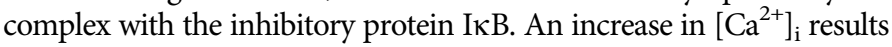
in I $\mathrm{B}$ degradation by ubiquitination, which is triggered upon the $\mathrm{Ca}^{2+}$-dependent phosphorylation of I $\mathrm{B}$. As a consequence, the p65 $\mathrm{NF}-\kappa \mathrm{B}$ subunit is released from I $\kappa \mathrm{B}$ inhibition and translocates into the nucleus (58) where it induces the expression of multiple proangiogenic genes (40). Consistently, we found that optical excitation significantly boosted the nuclear translocation of p65 in ECFCs cultured on the conjugated polymer compared with those not exposed to light. Robust up-regulation of several angiogenic genes, such as ICAM, SELE, MMP1, MMP2, and MMP9, which are under NF- $\kappa B-$ dependent transcriptional control, was also consequently observed. Intriguingly, $\mathrm{NF}-\kappa \mathrm{B}$ also mediates VEGFA-induced gene expression and angiogenesis in vascular endothelial cells $(59,60)$ through an increase in $\left[\mathrm{Ca}^{2+}\right]_{\mathrm{i}}$ (61). These observations strongly hint at NF- $\mathrm{KB}$ as the $\mathrm{Ca}^{2+}$-sensitive decoder that translates optical excitation into an angiogenic response in human ECFCs interfaced with the light-sensitive conjugated polymer.

Overall, our findings represent the proof of principle that optical modulation may be successfully exploited to directly control the fate of a progenitor cell population, i.e., ECFCs, which has been shown to support revascularization of ischemic tissues. The in vitro activation of ECFC angiogenic activity is made possible by the use of a biocompatible, light-sensitive polymer as the phototransduction element.

The combined use of optical excitation and organic polymer technology can open interesting perspectives for several different reasons. First, the use of light modulation allows unprecedented spatial and temporal resolution to be achieved in a fully reversible way. Light temporal and spatial patterns can be specifically designed and adapted to different in vitro cell models, allowing ideally endless combinations of possibilities, to finely tune overall output in cell proliferation and network formation. The demonstrated technology is minimally invasive, allows for massive parallelization of experiments, and can be virtually implemented in any cell therapy model in a straightforward way. In addition, the use of different polymers, with lower energy gap and in the form of nanobeads, may pave the way to the optical enhancement of therapeutic angiogenesis in vivo. Further work is needed to understand whether the pattern and/or intensity of the illumination protocol may be adjusted to further boost the angiogenic response. For instance, the optical excitation protocol consisted of 30-ms-long light pulses that were delivered at $1 \mathrm{~Hz}$ for 4 (tubulogenesis) up to 36 (proliferation) hours. This is likely to result in oscillations in $\left[\mathrm{Ca}^{2+}\right]_{\mathrm{i}}$, which are known to deliver the most instructive signal for ECFCs to undergo angiogenesis by inducing the nuclear translocation of the p65 NF- $\mathrm{KB}$ subunit (26). As the frequency of intracellular $\mathrm{Ca}^{2+}$ oscillations can be artificially manipulated to regulate NF- $\mathrm{KB}$-dependent gene expression in virtually any cell type (62), we envisage an additional layer of specificity and control that could be exploited to further improve the angiogenic response to optical excitation. Future work will also be devoted to assess the outcome of optical modulation on patient-derived ECFCs. One of the main hurdles associated to autologous cell-based therapy is the impairment of the angiogenic activity of EPCs, including ECFCs harvested from cardiovascular patients (29). The therapeutic translation of our findings will require the demonstration that light-induced TRPV1 activation boosts angiogenesis also in ECFCs derived from individuals affected by severe cardiovascular disorders, such as hypertension, atherosclerosis, 
and heart failure. In this view, the combination of organic semiconductors and genetic manipulation to increase endogenous TRPV1 expression could be sufficient to restore the reparative phenotype of autologous ECFCs from cardiovascular patients.

\section{MATERIALS AND METHODS \\ Semiconducting polymer and electrically insulating photoresist preparation}

Regioregular P3HT (99.995\% purity; Mn 54,000 to 75,000 molecular weight) was purchased from Sigma-Aldrich and used without any further purification. The samples for cell cultures were prepared by spin coating on a square $18 \mathrm{~mm}$ by $18 \mathrm{~mm}$ glass (VWR International) substrates carefully rinsed in subsequent ultrasonic baths of ultrapure water, acetone, and isopropanol. P3HT solution was prepared in chlorobenzene at a final P3HT concentration of $20 \mathrm{~g} /$ liter and spin coated on the cleaned substrates with a two-step recipe: (i) $3 \mathrm{~s}$ at $800 \mathrm{rpm}$ and (ii) $60 \mathrm{~s}$ at $1600 \mathrm{rpm}$. Polymer film thickness is about $150 \mathrm{~nm}$.

Microposit S1813 photoresist was purchased from Shipley and used without any further purification. Photoresist thin films were prepared by spin coating on cleaned substrates with a two-step recipe: (i) $3 \mathrm{~min}$ at $300 \mathrm{rpm}$ and (ii) $30 \mathrm{~s}$ at $2600 \mathrm{rpm}$. Parameters were adjusted to obtain homogeneous films and similar optical absorbance to the one of the polymer thin films, at the same excitation wavelength used in the long-term stimulation protocol (see below). All films were thermally treated in an oven at $120^{\circ} \mathrm{C}$ for 2 hours for annealing and sterilization. To promote adhesion, samples were coated with fibronectin (from bovine plasma; Sigma-Aldrich) at a concentration of $2 \mathrm{mg} / \mathrm{ml}$ in phosphate-buffered saline (PBS) for at least $30 \mathrm{~min}$ at $37^{\circ} \mathrm{C}$ and then rinsed with PBS.

\section{Isolation and cultivation of ECFCs}

ECFCs were isolated from peripheral blood and expanded as shown elsewhere (26). Blood samples (40 ml) collected in EDTA-containing tubes were obtained from healthy male human volunteers aged from 28 to 38 years. The Institutional Review Board at Istituto di Ricovero e Cura a Carattere Scientifico Policlinico San Matteo Foundation in Pavia approved all protocols and specifically approved this study. Informed written consent was obtained according to the Declaration of Helsinki of 1975 as revised in 2008 . We focused on the so-called ECFCs, a subgroup of EPCs that are found in the CD $34^{+} \mathrm{CD} 45^{-}$fraction of circulating mononuclear cells (MNCs), exhibit robust proliferative potential, and form capillary-like structures in vitro (23). To isolate ECFCs, MNCs were obtained from peripheral blood by density gradient centrifugation on lymphocyte separation medium for $30 \mathrm{~min}$ at $400 \mathrm{~g}$ and washed twice in EBM-2 with $2 \%$ fetal calf serum. A median of $36 \times 10^{6} \mathrm{MNCs}$ (range, 18 to 66 ) was plated on fibronectincoated culture dishes (BD Biosciences) in the presence of the endothelial cell growth medium EGM-2 MV (Lonza) containing endothelial basal medium (EBM-2), 5\% fetal bovine serum (FBS), recombinant human (rh) EGF, rhVEGF, recombinant human Fibroblast Growth Factor-Basic (rhFGF-B), recombinant human Insulin-like Growth Factor-1 (rhIGF-1), ascorbic acid, and heparin and maintained at $37^{\circ} \mathrm{C}$ in $5 \% \mathrm{CO}_{2}$ and humidified atmosphere. Nonadherent cells were discarded after 2 days, and thereafter, medium was changed three times a week. The outgrowth of ECFCs from adherent MNCs was characterized by the formation of a cluster of cobblestone-shaped cells. That ECFC-derived colonies belonged to the endothelial lineage was confirmed by staining with anti-CD31, anti-CD105, anti-
CD144, anti-CD146, anti-von Willebrand factor, anti-CD45, and anti-CD14 monoclonal antibodies and by assessment of capillarylike network formation in the in vitro tube formation assay.

For our experiments, we have mainly used endothelial cells obtained from early-passage ECFCs (P2-4, which roughly encompasses a 15- to 18-day period) with the purpose to avoid, or maximally reduce, any potential bias due to cell differentiation. However, to make sure that the phenotype of the cells did not change throughout the experiments, in the preliminary experiments, we tested the immunophenotype of ECFCs at different passages, and we found no differences. We also tested whether functional differences occurred when early (P2)- and late (P6)-passage ECFCs were used by testing the in vitro capacity of capillary network formation in a Cultrex assay and found no differences between early- and late-passage ECFC-derived cells (data not shown).

\section{Electrophysiology}

Electrophysiological recordings were performed using a patch-clamp setup (Axopatch 200B; Axon Instruments) coupled to an inverted microscope (Nikon Eclipse Ti). ECFCs were measured in whole-cell configuration with freshly pulled glass pipettes ( 3 to $6 \mathrm{M} \Omega$ ), filled with the following intracellular solution: $12 \mathrm{mM} \mathrm{KCl}, 125 \mathrm{mM}$ K-gluconate, $1 \mathrm{mM} \mathrm{MgCl} 2,0.1 \mathrm{mM} \mathrm{CaCl}_{2}, 10 \mathrm{mM}$ EGTA, $10 \mathrm{mM}$ Hepes, and $10 \mathrm{mM}$ ATP (adenosine 5'-triphosphate)- $\mathrm{Na}_{2}$. The extracellular solution contained the following: $135 \mathrm{mM} \mathrm{NaCl}, 5.4 \mathrm{mM} \mathrm{KCl}, 5 \mathrm{mM}$ Hepes, $10 \mathrm{mM}$ glucose, $1.8 \mathrm{mM} \mathrm{CaCl}_{2}, 1 \mathrm{mM} \mathrm{MgCl}_{2}$. Only single cells were selected for recordings. Acquisition was performed with the pCLAMP 10 software (Axon Instruments). Membrane currents were low pass filtered at $2 \mathrm{kHz}$ and digitized with a sampling rate of $10 \mathrm{kHz}$ (Digidata 1440 A; Molecular Devices). Data were analyzed with Clampfit (Axon Instruments) and Origin 8.0 (OriginLab Corporation).

\section{Polymer photoexcitation}

For optical excitation of the polymer, a homemade petri cell culture illuminator, compatible with the use within the cell incubator, was designed and implemented. Its design included a black spacer made by fused filament fabrication, both to minimize overheating effects in the extracellular bath and to avoid unwanted light scattering/diffusion effects and cross-talk between different specimens. Optical excitation was provided by a green LED system, whose duty cycle, repetition rate, and intensity were set through a custom-made control circuit, comprising a microcontroller, a digital-to-analog converter, and an analog LED driver. The driver was connected to five green LEDs (SMB1N-525V-02; Roithner LaserTechnik GmbH, Vienna, Austria), with maximum emission wavelength at $525 \mathrm{~nm}$, each carrying a collimator lens reducing the emission angle to $22^{\circ}$. This way, up to five $3.5-\mathrm{cm}$ petri dishes can be simultaneously treated with a homogeneous photoexcitation density of $40 \mathrm{~mW} / \mathrm{cm}^{2}$. The long-term optical excitation protocol adopted for cell fate modulation consists of 30-ms-long pulses, followed by 70 -ms-long dark conditions, continuously repeated for a minimum of 4 up to 36 hours in the case of tubulogenesis and proliferation assays, respectively.

\section{Proliferation assays}

Growth dynamics were evaluated by plating a total of $5 \times 10^{3}$ ECFCderived cells into $10-\mathrm{mm}$ fibronectin-treated cloning cylinders $(5 \times$ $10^{4} / \mathrm{cm}^{2}$ ) in the presence of EGM-2 MV medium to facilitate the adhesion. After 12 hours, the medium was switched to EBM-2 supplemented with $2 \%$ fetal calf serum. For the pharmacological treatment, one of compounds was added to the medium: BAPTA $(30 \mu \mathrm{M}), \mathrm{CPZ}$ $(10 \mu \mathrm{M}), \mathrm{RN}-1734(20 \mu \mathrm{M})$, or RR $(10 \mu \mathrm{M})$. Cultures were incubated 
at $37^{\circ} \mathrm{C}$ (in $5 \% \mathrm{CO}_{2}$ and humidified atmosphere), and cell growth was assessed after 36 hours since the beginning of the long-term illumination protocol. At this point, cells were recovered by trypsinization from all the dishes, and the cell number was assessed by counting in a hemocytometer. Preliminary experiments showed no unspecific or toxic effect for each agent when used at these concentrations. Each assay was repeated in triplicate.

\section{In vitro tube formation assay}

ECFC-derived cells from early-passage (P2 to P4) cultures were obtained by trypsinization and resuspended in EBM-2 supplemented with $2 \%$ FBS. EPC-derived cells $\left(10 \times 10^{3}\right)$ per well were plated in Cultrex basement membrane extract (Trevigen Inc., Gaithersburg, MD, USA) 10 -mm fibronectin-treated cloning cylinders. Plates were then incubated at $37^{\circ} \mathrm{C}, 5 \% \mathrm{CO}_{2}$, and capillary network formation was assessed starting from 4 to 24 hours later. At least three different sets of cultures were performed every experimental point. Quantification of tubular structures was performed after 8 hours of incubation by measuring the total length of structures per field with the aid of the ImageJ software (National Institutes of Health, USA; http://rsbweb.nih.gov/ij/). To evaluate the role of TRPV1, the same protocol was repeated in the presence of the following drugs: BAPTA $(30 \mu \mathrm{M}), \mathrm{CPZ}(10 \mu \mathrm{M}), \mathrm{RN}-1734$ $(20 \mu \mathrm{M})$, or RR $(10 \mu \mathrm{M})$.

\section{ROS detection}

$\mathrm{H}_{2}$ DCF-DA (Sigma-Aldrich) was used for the intracellular detection of ROS. ECFCs were seeded onto polymer and control substrates and subjected to the same photoexcitation protocol used for the in vitro tube formation assay. Immediately after the end of the protocol, cell cultures were incubated with the ROS probe for $30 \mathrm{~min}$. After careful washout of the excess probe from the extracellular medium, the fluorescence of the probe was recorded (excitation/emission wavelengths, $490 / 520 \mathrm{~nm}$; integration time, $70 \mathrm{~ms}$ for $\mathrm{H}_{2}$ DCF-DA) with an inverted microscope (Nikon Eclipse Ti) equipped with an Analog-WDM Camera (CoolSNAP MYO, Teledyne Photometrics). To minimize the effects of the spectral overlap between the polymer absorption and emission spectra, and the probe emission, samples were turned upside down by using a homemade chamber with a $500-\mu \mathrm{m}$-thick channel filled with extracellular medium. Variation of fluorescence intensity was evaluated over regions of interest covering single-cell areas, and reported values represent the average over multiple cells. See figure captions for additional details about statistical analysis. Image processing was carried out with ImageJ and subsequently analyzed with Origin 8.0.

\section{Polymer photostability assay}

Two sets of P3HT thin films $(n=12)$ were prepared as described above. The optical absorbance, the emission, and the Raman spectrum were measured immediately after fabrication. Then, all samples were exposed to ECFC growth medium (EBM-2 supplemented with 2\% FBS) and incubated at $37^{\circ} \mathrm{C}, 5 \% \mathrm{CO}_{2}$ for 24 hours. The first set was taken in dark conditions $(n=6)$, and the second one was treated with the same optical excitation protocol used in the tubulogenesis assays $(n=6)$. After incubation, absorption, emission, and Raman spectrum were measured again in the same conditions as before. Absorption spectra were recorded by using a spectrophotometer (PerkinElmer Lambda 1040) in transmission mode. Photoluminescence spectra were acquired by using a Jobin-Yvon spectrofluorometer; the excitation wavelength was set at the polymer absorption peak wavelength $(530 \mathrm{~nm})$. Reso- nant Raman spectra were recorded by using visible light excitation at $532 \mathrm{~nm}$ (HORIBA Jobin-Yvon HR800 micro-Raman spectrometer system). Laser power intensity on the sample was kept at values lower than $0.03 \mathrm{~mW}$ to avoid laser-induced sample degradation. Spectra were typically recorded in the region 600 to $2000 \mathrm{~cm}^{-1}$ and were calibrated against the $520.5 \mathrm{~cm}^{-1}$ line of an internal silicon wafer. The signal-to-noise ratio was enhanced by repeated acquisitions (100). The measurements were conducted at room temperature (RT), and the resulting spectral resolution was $0.4 \mathrm{~cm}^{-1}$.

\section{Immunofluorescence assay}

To examine NF- $\kappa \mathrm{B}$ p 65 subunit translocation into the nucleus in the individual ECFCs, the coverslips were fixed with $4 \%$ formaldehyde in PBS (20 min at RT) and permeabilized with $0.1 \%$ Triton X-100 in PBS (7 min at RT). Primary rabbit polyclonal anti-p65 antibody (Santa Cruz Biotechnology, catalog no. Sc-372) was applied at a final dilution of $1: 100$ for 1 hour at $37^{\circ} \mathrm{C}$ in a humidified chamber. After three washes with PBS, secondary chicken anti-rabbit Alexa(488)conjugated antibody (1:200; Invitrogen, catalog no. A-21441) was applied for 1 hour at RT. After washing (three times in PBS), nuclei were counterstained with 4',6-diamidino-2-phenylindole, dihydrochloride (DAPI; 1:5000 dilution in PBS; 20 min at RT; Invitrogen, catalog no. D1306). Last, the coverslips with cells were mounted on microscope glass slides using Fluoroshield mount medium (Sigma, catalog no. F6182). Fluorescence images were taken with the same fluorescence microscope used for the electrophysiology experiments, using standard DAPI and fluorescein isothiocyanate filters set for the acquisition of DAPI and Alexa(488) fluorescence emission, respectively.

\section{Total RNA extraction and real-time polymerase chain reaction}

Cells were lysed in $0.5 \mathrm{ml}$ of TRI Reagent (Sigma, catalog no. T9424), and total RNA was extracted according to the manufacturer's protocol. One microgram of total RNA was retrotranscribed using SensiFAST cDNA Synthesis Kit (Bioline, London, UK, catalog no. BIO-65054). Real-time polymerase chain reaction (PCR) was performed using iTaq qPCR master mix according to the manufacturer's instructions (Bio-Rad, Segrate, Italy, catalog no. 1725124) on a SFX96 Real-Time System (BioRad). As a control, $S 18$ ribosomal subunit was used, whose expression did not change across the conditions. For each gene, $\Delta C_{\mathrm{t}}$ was calculated by using the formula $\Delta C_{\mathrm{t}}=2^{\wedge}\left(\Delta C_{\mathrm{t}}\right.$ (gene $\left.)-\Delta C_{\mathrm{t}}(\mathrm{S} 18)\right)$. The data are expressed as a percentage variation between $\mathrm{P} 3 \mathrm{HT}$ light and glass light conditions and P3HT dark and glass dark samples, respectively. Sequences of oligonucleotide primers are listed in table S1.

\section{Statistical analysis}

The significance of differences was evaluated with unpaired Student's $t$ test or one-way analysis of variance (ANOVA) coupled with Tukey or Dunnett's post hoc test, as appropriate. Data are represented as means \pm standard error of the mean (SEM). $P<0.05$ was considered statistically significant. Statistical analysis was performed using the GraphPad Prism 7 software (GraphPad Software Inc., La Jolla, CA).

\section{SUPPLEMENTARY MATERIALS}

Supplementary material for this article is available at http://advances.sciencemag.org/cgi/ content/full/5/9/eaav4620/DC1

Fig. S1. Local and global evaluation of the extracellular bath temperature.

Fig. S2. TRPV1 is endogenously expressed in ECFCs, and it is efficiently activated by polymer photostimulation. 
Fig. S3. Current clamp measurements in HEK-293 cells.

Fig. S4. Pharmacological study on ECFCs seeded on polymer substrates in the dark-Evaluation of effect on tubulogenesis.

Fig. S5. Polymer photostability.

Fig. S6. p65 NF-кB nuclear translocation is unaltered in ECFCs seeded on glass subjected to light-induced photostimulation.

Fig. S7. mRNA levels of proangiogenic genes downstream of NF- $\mathrm{KB}$ signaling.

Table S1. List of oligonucleotide primers used for real-time PCR.

\section{REFERENCES AND NOTES}

1. J. Rivnay, S. Inal, B. A. Collins, M. Sessolo, E. Stavrinidou, X. Strakosas, C. Tassone, D. M. Delongchamp, G. G. Malliaras, Structural control of mixed ionic and electronic transport in conducting polymers. Nat. Commun. 7, 11287 (2016).

2. A. Campana, T. Cramer, D. T. Simon, M. Berggren, F. Biscarini, Electrocardiographic recording with conformable organic electrochemical transistor fabricated on resorbable bioscaffold. Adv. Mater. 26, 3874-3878 (2014).

3. M. Y. Mulla, E. Tuccori, M. Magliulo, G. Lattanzi, G. Palazzo, K. Persaud, L. Torsi, Capacitance-modulated transistor detects odorant binding protein chiral interactions. Nat. Commun. 6, 6010 (2015).

4. M. Sytnyk, M. Jakešová, M. Litviňuková, O. Mashkov, D. Kriegner, J. Stangl, J. Nebesářová, F. W. Fecher, W. Schöfberger, N. S. Sariciftci, R. Schindl, W. Heiss, E. D. Głowacki, Cellular interfaces with hydrogen-bonded organic semiconductor hierarchical nanocrystals. Nat. Commun. 8, 91 (2017).

5. M. R. Antognazza, M. D. Paolo, D. Ghezzi, M. Mete, S. D. Marco, J. F. Maya-Vetencourt, R. Maccarone, A. Desii, F. D. Fonzo, M. Bramini, A. Russo, L. Laudato, I. Donelli, M. Cilli, G. Freddi, G. Pertile, G. Lanzani, S. Bisti, F. Benfenati, Characterization of a polymer-based, fully organic prosthesis for implantation into the subretinal space of the rat. Adv. Healthc. Mater. 5, 2271-2282 (2016).

6. F. Di Maria, F. Lodola, E. Zucchetti, F. Benfenati, G. Lanzani, The evolution of artificial light actuators in living systems: From planar to nanostructured interfaces. Chem. Soc. Rev. 47, 4757-4780 (2018).

7. J. Rivnay, S. Inal, A. Salleo, R. M. Owens, M. Berggren, G. G. Malliaras, Organic electrochemical transistors. Nat. Rev. Mater. 3, 17086 (2018).

8. D. T. Simon, E. O. Gabrielsson, K. Tybrandt, M. Berggren, Organic bioelectronics: Bridging the signaling gap between biology and technology. Chem. Rev. 116, 13009-13041 (2016).

9. T. Someya, Z. Bao, G. G. Malliaras, The rise of plastic bioelectronics. Nature 540, 379-385 (2016).

10. J. F. Maya-Vetencourt, D. Ghezzi, M. R. Antognazza, E. Colombo, M. Mete, P. Feyen, A. Desii, A. Buschiazzo, M. D. Paolo, S. D. Marco, F. Ticconi, L. Emionite, D. Shmal, C. Marini, I. Donelli, G. Freddi, R. Maccarone, S. Bisti, G. Sambuceti, G. Pertile, G. Lanzani, F. Benfenati, A fully organic retinal prosthesis restores vision in a rat model of degenerative blindness. Nat. Mater. 16, 681-689 (2017).

11. D. Rand, M. Jakešová, G. Lubin, I. Vèbraite, M. David-Pur, V. Đerek, T. Cramer, N. S. Sariciftci, Y. Hanein, E. D. Głowacki, Direct electrical neurostimulation with organic pigment photocapacitors. Adv. Mater. 30, e1707292 (2018).

12. Y. Takano, T. Numata, K. Fujishima, K. Miyake, K. Nakao, W. D. Grove, R. Inoue, M. Kengaku, S. Sakaki, Y. Mori, T. Murakami, H. Imahori, Optical control of neuronal firing via photoinduced electron transfer in donor-acceptor conjugates. Chem. Sci. 7, 3331-3337 (2016).

13. N. Martino, P. Feyen, M. Porro, C. Bossio, E. Zucchetti, D. Ghezzi, F. Benfenati, G. Lanzani, M. R. Antognazza, Photothermal cellular stimulation in functional bio-polymer interfaces. Sci. Rep. 5, 8911 (2015).

14. P. Feyen, E. Colombo, D. Endeman, M. Nova, L. Laudato, N. Martino, M. R. Antognazza, G. Lanzani, F. Benfenati, D. Ghezzi, Light-evoked hyperpolarization and silencing of neurons by conjugated polymers. Sci. Rep. 6, 22718 (2016).

15. C. Tortiglione, M. R. Antognazza, A. Tino, C. Bossio, V. Marchesano, A. Bauduin, M. Zangoli, S. V. Morata, G. Lanzani, Semiconducting polymers are light nanotransducers in eyeless animals. Sci. Adv. 3, e1601699 (2017).

16. G.-J. Jeong, J. Y. Oh, Y.-J. Kim, S. H. Bhang, H.-K. Jang, J. Han, J.-K. Yoon, S.-M. Kwon, T. I. Lee, B.-S. Kim, Therapeutic angiogenesis via solar cell-facilitated electrical stimulation. ACS Appl. Mater. Interfaces 9, 38344-38355 (2017).

17. K. Yang, J. Y. Oh, J. S. Lee, Y. Jin, G.-E. Chang, S. S. Chae, E. Cheong, H. K. Baik, S.-W. Cho, Photoactive poly(3-hexylthiophene) nanoweb for optoelectrical stimulation to enhance neurogenesis of human stem cells. Theranostics 7, 4591-4604 (2017).

18. A. Savchenko, V. Cherkas, C. Liu, G. B. Braun, A. Kleschevnikov, Y. I. Miller, E. Molokanova, Graphene biointerfaces for optical stimulation of cells. Sci. Adv. 4, eaat0351 (2018).

19. A. Liebert, A. Krause, N. Goonetilleke, B. Bicknell, H. Kiat, A role for photobiomodulation in the prevention of myocardial ischemic reperfusion injury: A systematic review and potential molecular mechanisms. Sci. Rep. 7, 42386 (2017).
20. I.-S. Park, P.-S. Chung, J. C. Ahn, Adipose-derived stromal cell cluster with light therapy enhance angiogenesis and skin wound healing in mice. Biochem. Biophys. Res. Commun. 462, 171-177 (2015).

21. S. Rohringer, W. Holnthoner, S. Chaudary, P. Slezak, E. Priglinger, M. Strassl, K. Pill, S. Mühleder, H. Redl, P. Dungel, The impact of wavelengths of LED light-therapy on endothelial cells. Sci. Rep. 7, 10700 (2017).

22. J. E. Pomeroy, H. X. Nguyen, B. D. Hoffman, N. Bursac, Genetically encoded photoactuators and photosensors for characterization and manipulation of pluripotent stem cells. Theranostics 7, 3539-3558 (2017).

23. D. A. Ingram, L. E. Mead, H. Tanaka, V. Meade, A. Fenoglio, K. Mortell, K. Pollok, M. J. Ferkowicz, D. Gilley, M. C. Yoder, Identification of a novel hierarchy of endothelial progenitor cells using human peripheral and umbilical cord blood. Blood 104, 2752-2760 (2004)

24. D. Tasev, P. Koolwijk, V. W. M. van Hinsbergh, Therapeutic potential of human-derived endothelial colony-forming cells in animal models. Tissue Eng. Part B Rev. 22, 371-382 (2016).

25. S. Fraineau, C. G. Palii, B. M. Neill, M. Ritso, W. C. Shelley, N. Prasain, A. Chu, E. Vion, K. Rieck, S. Nilufar, T. J. Perkins, M. A. Rudnicki, D. S. Allan, M. C. Yoder, E. J. Suuronen, M. Brand, Epigenetic activation of pro-angiogenic signaling pathways in human endothelial progenitors increases vasculogenesis. Stem Cell Rep. 9, 1573-1587 (2017).

26. S. Dragoni, U. Laforenza, E. Bonetti, F. Lodola, C. Bottino, R. Berra-Romani, G. C. Bongio, M. P. Cinelli, G. Guerra, P. Pedrazzoli, V. Rosti, F. Tanzi, F. Moccia, Vascular endothelial growth factor stimulates endothelial colony forming cells proliferation and tubulogenesis by inducing oscillations in intracellular $\mathrm{Ca}^{2+}$ concentration. Stem Cells 29, 1898-1907 (2011).

27. F. Lodola, U. Laforenza, F. Cattaneo, F. A. Ruffinatti, V. Poletto, M. Massa, R. Tancredi, E. Zuccolo, D. A. Khdar, A. Riccardi, M. Biggiogera, V. Rosti, G. Guerra, F. Moccia, VEGF-induced intracellular $\mathrm{Ca}^{2+}$ oscillations are down-regulated and do not stimulate angiogenesis in breast cancer-derived endothelial colony forming cells. Oncotarget $\mathbf{8}$, 95223-95246 (2017).

28. F. Lodola, U. Laforenza, E. Bonetti, D. Lim, S. Dragoni, C. Bottino, H. L. Ong, G. Guerra, C. Ganini, M. Massa, M. Manzoni, I. S. Ambudkar, A. A. Genazzani, V. Rosti, P. Pedrazzoli, F. Tanzi, F. Moccia, C. Porta, Store-operated $\mathrm{Ca}^{2+}$ entry is remodelled and controls in vitro angiogenesis in endothelial progenitor cells isolated from tumoral patients. PLOS ONE 7, e42541 (2012).

29. F. Moccia, R. Berra-Romani, V. Rosti, Manipulating intracellular $\mathrm{Ca}^{2+}$ signals to stimulate therapeutic angiogenesis in cardiovascular disorders. Curr. Pharm. Biotechnol. 19, 686-699 (2018).

30. V. Benfenati, N. Martino, M. R. Antognazza, A. Pistone, S. Toffanin, S. Ferroni, G. Lanzani, M. Muccini, Photostimulation of whole-cell conductance in primary rat neocortical astrocytes mediated by organic semiconducting thin films. Adv. Healthc. Mater. 3, 392-399 (2014).

31. J. Yao, B. Liu, F. Qin, Rapid temperature jump by infrared diode laser irradiation for patch-clamp studies. Biophys. J. 96, 3611-3619 (2009).

32. N. A. Hofmann, S. Barth, M. Waldeck-Weiermair, C. Klec, D. Strunk, R. Malli, W. F. Graier, TRPV1 mediates cellular uptake of anandamide and thus promotes endothelial cell proliferation and network-formation. Biol. Open 3, 1164-1172 (2014).

33. F. Lodola, N. Martino, G. Tullii, G. Lanzani, M. R. Antognazza, Conjugated polymers mediate effective activation of the mammalian ion channel transient receptor potential vanilloid 1. Sci. Rep. 7, 8477 (2017).

34. S. Dragoni, G. Guerra, A. F. Pla, G. Bertoni, A. Rappa, V. Poletto, C. Bottino, A. Aronica, F. Lodola, M. P. Cinelli, U. Laforenza, V. Rosti, F. Tanzi, L. Munaron, F. Moccia, A functional transient receptor potential vanilloid 4 (TRPV4) channel is expressed in human endothelial progenitor cells: TRPV4 expression in EPCs. J. Cell. Physiol. 230, 95-104 (2015)

35. A. B. Parekh, $\mathrm{Ca}^{2+}$ microdomains near plasma membrane $\mathrm{Ca}^{2+}$ channels: Impact on cell function. J. Physiol. 586, 3043-3054 (2008).

36. M. Plaksin, E. Shapira, E. Kimmel, S. Shoham, Thermal transients excite neurons through universal intramembrane mechanoelectrical effects. Phys. Rev. X 8, 011043 (2018).

37. C. Bossio, I. A. Aziz, G. Tullii, E. Zucchetti, D. Debellis, M. Zangoli, F. D. Maria, G. Lanzani, M. R. Antognazza, Photocatalytic activity of polymer nanoparticles modulates intracellular calcium dynamics and reactive oxygen species in HEK-293 cells. Front. Bioeng. Biotechnol. 6, 114 (2018).

38. G. Tullii, A. Desii, C. Bossio, S. Bellani, M. Colombo, N. Martino, M. R. Antognazza, G. Lanzani, Bimodal functioning of a mesoporous, light sensitive polymer/electrolyte interface. Org. Electron. 46, 88-98 (2017).

39. S. Bellani, A. Ghadirzadeh, L. Meda, A. Savoini, A. Tacca, G. Marra, R. Meira, J. Morgado, F. D. Fonzo, M. R. Antognazza, Hybrid organic/inorganic nanostructures for highly sensitive photoelectrochemical detection of dissolved oxygen in aqueous media. Adv. Funct. Mater. 25, 4531-4538 (2015).

40. S. P. Tabruyn, A. W. Griffioen, NF-кB: A new player in angiostatic therapy. Angiogenesis 11, 101-106 (2008). 
41. B. A. Tompkins, W. Balkan, J. Winkler, M. Gyöngyösi, G. Goliasch, F. Fernández-Avilés, J. M. Hare, Preclinical studies of stem cell therapy for heart disease. Circ. Res. 122, 1006-1020 (2018).

42. E. B. Peters, Endothelial progenitor cells for the vascularization of engineered tissues. Tissue Eng. Part B Rev. 24, 1-24 (2018).

43. S. Bevan, T. Quallo, D. A. Andersson, TRPV1. Handb. Exp. Pharmacol. 222, 207-245 (2014).

44. D. J. DelloStritto, P. J. Connell, G. M. Dick, I. S. Fancher, B. Klarich, J. N. Fahmy, P. T. Kang, Y.-R. Chen, D. S. Damron, C. K. Thodeti, I. N. Bratz, Differential regulation of TRPV1 channels by $\mathrm{H}_{2} \mathrm{O}_{2}$ : Implications for diabetic microvascular dysfunction. Basic Res. Cardiol. 111, 21 (2016).

45. Y.-J. Lin, H.-H. Hsu, T. Ruan, Y. R. Kou, Mediator mechanisms involved in TRPV1, TRPA1 and $\mathrm{P} 2 \mathrm{X}$ receptor-mediated sensory transduction of pulmonary ROS by vagal lung C-fibers in rats. Respir. Physiol. Neurobiol. 189, 1-9 (2013).

46. T. Ruan, Q. Gu, Y. R. Kou, L.-Y. Lee, Hyperthermia increases sensitivity of pulmonary C-fibre afferents in rats: Hyperthermia enhances pulmonary C-fibre excitability. J. Physiol. 565, 295-308 (2005).

47. I. da Silva, M. Barroso, T. Moura, R. Castro, G. Soveral, Endothelial aquaporins and hypomethylation: Potential implications for atherosclerosis and cardiovascular disease. Int. J. Mol. Sci. 19, E130 (2018).

48. S. Martinotti, U. Laforenza, M. Patrone, F. Moccia, E. Ranzato, Honey-mediated wound healing: $\mathrm{H}_{2} \mathrm{O}_{2}$ entry through AQP3 determines extracellular $\mathrm{Ca}^{2+}$ influx. Int. J. Mol. Sci. 20, 764 (2019).

49. J. P. M. White, M. Cibelli, L. Urban, B. Nilius, J. G. McGeown, I. Nagy, TRPV4: Molecular conductor of a diverse orchestra. Physiol. Rev. 96, 911-973 (2016)

50. M. Suzuki, A. Mizuno, K. Kodaira, M. Imai, Impaired pressure sensation in mice lacking TRPV4. J. Biol. Chem. 278, 22664-22668 (2003).

51. E. Panieri, M. M. Santoro, ROS signaling and redox biology in endothelial cells. Cell. Mol. Life Sci. 72, 3281-3303 (2015).

52. K.-H. Su, S.-J. Lin, J. Wei, K.-I. Lee, J.-F. Zhao, S.-K. Shyue, T.-S. Lee, The essential role of transient receptor potential vanilloid 1 in simvastatin-induced activation of endothelial nitric oxide synthase and angiogenesis. Acta Physiol. 212, 191-204 (2014).

53. Y.-H. Yang, H. Zhou, N. O. Binmadi, P. Proia, J. R. Basile, Plexin-B1 activates NF-kB and IL-8 to promote a pro-angiogenic response in endothelial cells. PLOS ONE 6, e25826 (2011).

54. T. H. Lee, H. Jung, K. H. Park, M. H. Bang, N.-I. Baek, J. Kim, Jaceosidin, a natural flavone, promotes angiogenesis via activation of VEGFR2/FAK/PI3K/AKT/NF-KB signaling pathways in endothelial cells. Exp. Biol. Med. 239, 1325-1334 (2014).

55. M. Muller, A. Morotti, C. Ponzetto, Activation of NF- B is essential for hepatocyte growth factor-mediated proliferation and tubulogenesis. Mol. Cell. Biol. 22, 1060-1072 (2002).

56. C. Li, L. Bo, Q. Liu, W. Liu, X. Chen, D. Xu, F. Jin, Activation of TRPV1-dependent calcium oscillation exacerbates seawater inhalation-induced acute lung injury. Mol. Med. Rep. 13, 1989-1998 (2016).

57. F. A. Pinho-Ribeiro, M. S. N. Hohmann, S. M. Borghi, A. C. Zarpelon, C. F. S. Guazelli, M. F. Manchope, R. Casagrande, W. A. Verri Jr., Protective effects of the flavonoid hesperidin methyl chalcone in inflammation and pain in mice: Role of TRPV1, oxidative stress, cytokines and NF-кB. Chem. Biol. Interact. 228, 88-99 (2015).
58. D. Lim, A. lyer, V. Ronco, A. A. Grolla, P. L. Canonico, E. Aronica, A. A. Genazzani, Amyloid beta deregulates astroglial mGluR5-mediated calcium signaling via calcineurin and Nf-kB. Glia 61, 1134-1145 (2013).

59. I. Kim, S.-O. Moon, S. H. Kim, H. J. Kim, Y. S. Kohand, G. Y. Koh, Vascular endothelial growth factor expression of intercellular adhesion molecule 1 (ICAM-1), vascular cell adhesion molecule 1 (VCAM-1), and E-selectin through nuclear factor- $\kappa B$ activation in endothelial cells. J. Biol. Chem. 276, 7614-7620 (2001).

60. J. Grosjean, S. Kiriakidis, K. Reilly, M. Feldmann, E. Paleolog, Vascular endothelial growth factor signalling in endothelial cell survival: A role for NFKB. Biochem. Biophys. Res. Commun. 340, 984-994 (2006).

61. D. P. Noren, W. H. Chou, S. H. Lee, A. A. Qutub, A. Warmflash, D. S. Wagner, A. S. Popel, A. Levchenko, Endothelial cells decode VEGF-mediated $\mathrm{Ca}^{2+}$ signaling patterns to produce distinct functional responses. Sci. Signal. 9, -ra20 (2016).

62. A. B. Parekh, Decoding cytosolic $\mathrm{Ca}^{2+}$ oscillations. Trends Biochem. Sci. 36, 78-87 (2011).

Acknowledgments: We gratefully thank I. Abdel Aziz for the characterization of the homemade petri cell culture illuminator used for long-term optical excitation and P. Falvo for the constructive criticism of the manuscript and the helpful scientific discussions. Funding: This work was jointly supported by the European Research Council (ERC) under the European Union's Horizon 2020 research and innovation program "LINCE," grant agreement no. 803621 (M.R.A.), the EU Horizon 2020 FETOPEN-2018-2020 Programme "LION-HEARTED," grant agreement no. 828984 (F.L., F.M., and M.R.A.), the Italian Ministry of Education, University and Research (MIUR): Dipartimenti di Eccellenza Program (2018-2022)_Department of Biology and Biotechnology "L. Spallanzani," University of Pavia (F.M.), and Fondo Ricerca Giovani from the University of Pavia (F.M.). Author contributions: F.L., F.M., and M.R.A. planned the experiments. F.L. carried out the experimental measurements (electrophysiology, short- and long-term photoexcitation, evaluation of effects on proliferation, tubulogenesis, and ROS production). V.R. provided the ECFC models, took care of the cell cultures, and contributed to the tubulogenesis and proliferation experiments. G.T. prepared the polymer samples. A.D. designed, realized, and optimized the experimental setup for the long-term photoexcitation. L.T. and D.L. carried out the immunofluorescence and real-time PCR assays. P.C. contributed to the methodological discussion about gene expression. F.L. and M.R.A. wrote the main manuscript, with help from F.M. All authors contributed to the data interpretation and approved the final manuscript. Competing interests: The authors declare that they have no competing interests. Data and materials availability: All data needed to evaluate the conclusions in the paper are present in the paper and/or the Supplementary Materials. Additional data related to this paper may be requested from the authors.

Submitted 20 September 2018

Accepted 4 September 2019

Published 27 September 2019

$10.1126 /$ sciadv.aav4620

Citation: F. Lodola, V. Rosti, G. Tullii, A. Desii, L. Tapella, P. Catarsi, D. Lim, F. Moccia, M. R. Antognazza, Conjugated polymers optically regulate the fate of endothelial colonyforming cells. Sci. Adv. 5, eaav4620 (2019). 